\title{
Evaluation of the Effects of Donepezil, Memantine and $\alpha$-lipoic Acid Combined Administration in Amnesia Rats on Impaired Cognitive Functions in Terms of Behavioural, Apoptotic, Cholinergic and Glutamatergic Systems
}

\author{
Asli Aykac $^{1}$, Dilek Ozbeyli² ${ }^{\circledR}$, Gizem Pekol ${ }^{\circledR}$, Ahmet Ozer Sehirli ${ }^{4}$ \\ ${ }^{1}$ Near East University, Department of Biophysics, Nicosia, Cyprus. \\ ${ }_{2}^{2}$ Marmara University, Vocational School of Health Services, Istanbul, Turkey. \\ ${ }^{3}$ Near East University, Faculty of Medicine, Nicosia, Cyprus. \\ ${ }^{4}$ Near East University, Faculty of Dentistry, Nicosia, Cyprus. \\ Correspondence Author: Asli Aykac \\ E-mail: asli.aykac@neu.edu.tr
}

Received: $08.01 .2021 \quad$ Accepted: 18.10 .2021

\begin{abstract}
Objective: The aim of the study was to evaluate the possible protective effect of donepezil, memantine and alpha-lipoic acid ( $\alpha$-LA) combined therapy in the scopolamine-induced amnesia rat model.

Methods: In this study, the effect of combined therapy used in the treatment of scopolamine-induced amnesia on behavioural parameters was evaluated using Y-maze and new object recognition (NOR) test. In addition, muscarinic acetylcholine receptor subtype M1, N-methyl-Daspartate receptor NR2B subunit, brain-derived neurotrophic factor (BDNF) and mitochondrial apoptosis-related proteins [B-cell lymphoma-2 ( $\mathrm{BCl}-2$ ) / Bcl-2 associated X (Bax) ratio, caspase (casp) - 3, and -9] expression levels were evaluated using the western blot method in the frontal cortex and hippocampus regions.

Results: The main findings of this study demonstrated that in scopolamine-induced amnesia rats, cognitive dysfunction determined by both the $Y$-maze and the NOR test were reversed with the combined treatment of memantine, donepezil and $\alpha$-LA. According to immunoblotting results in both brain regions, scopolamine-induced decreased M1, BDNF, Bcl-2 / Bax ratio and increased NR2B, casp-3 and - 9 expression levels were found to be reversed to almost control values with combined treatment.

Conclusion: Consistent with the literature, our study results suggest that the positive contribution of $\alpha$-LA to the combined treatment of donepezil and memantine, which is used in the routine treatment of neurodegenerative diseases, may be a treatment option in the future.

Keywords: Alpha-lipoic acid, amnesia, apoptosis, scopolamine, Y-maze.
\end{abstract}

\section{INTRODUCTION}

The incidence of Alzheimer's disease (AD), which is a neurodegenerative disease and one of the most common causes of amnesia, is increasing due to the prolongation of the average life expectancy. While a number of pathophysiological events such as the degeneration of cholinergic and glutaminergic neurons stand out in the pathophysiology of $A D$, the etiology of $A D$ has not been fully elucidated yet (1).

Cognitive disorders seen in $A D$ can be mimicked by creating amnesia caused by Scopolamine, a muscarinic acetylcholine receptor (mAChR) antagonist, in rodents (2). Scopolamineinduced amnesia, showing the possible interaction between mAChR and N-methyl-D-aspartate receptor (NMDAR) in the cognitive functions in the hippocampus and frontal cortex $(3,4)$. Brain-derived neurotrophic factor (BDNF) is an important component in the preservation of synaptic plasticity and synaptogenesis in the hippocampus region, which is the basis of memory and consolidation. While there are studies emphasizing the decrease of BDNF level and protein expression occurring in $A D$ process, which reveals the importance of $B D N F$ in $A D$ pathogenesis, there are also conflicting results in the literature $(5,6)$.

NMDARs carry out learning and memory activities in the brain, consist of NMDAR 1 and 2 (NR1, NR2) subunits. It has been reported that $A D$ causes degeneration of the hippocampus, cell loss, extensive pathological changes and increases in NR2 density due to cell death in the hippocampus and frontal cortex regions (7). While glutamate ligands can function as a general marker of NR2 subunits, it is almost impossible to conclude whether specific combinations of an NR2 subunit or all subunits have changed in $A D$. In the literature by using subunit-specific 
antibodies, it is reported that protein levels decrease for NR2B, not NR2A, throughout the hippocampus of $A D$ subjects with moderate and severe pathology (8). Studies show that the blockade of NMDAR function causes neuronal apoptosis and degeneration. On the other hand, excessive NMDAR activity in terms of the magnitude of activation has deleterious effects on neuronal structures. In the literature, memantine, (1-amino-3, 5-dimethyladamantane hydrochloride), a noncompetitive NMDAR antagonist, has been shown to reduce oxidative damage and prevent cellular aging in neuronal cells (9). The acetylcholinesterase inhibitor donepezil was developed to prevent a decrease in cholinergic conduction due to the display of cholinergic neuron degeneration of $A D$ (10). The existence of evidence for functional impairment of neurotransmitter systems in $A D$ is emphasized cholinergic activity findings especially in cortical projections in AD patients' cognitive deficiency. This case constitutes the basic rationale of cholinergic replacement therapy in the treatment approach of $A D$ patients. Despite the reported symptomatic and cognitive benefits of donepezil and memantine in $A D$ patients, these drugs cannot stop or prevent AD progression (11-13).

The disruption/damage occurring in the mitochondria, which plays a key role in the bioenergetic modulation of the cell, can lead to activation of the caspase cascade and thus cell death. In post-mortem research on AD patients' brains, it was found that there was an increase in mitochondrial DNA and proteins $(14,15) . \alpha-L A$ is a natural antioxidant synthesized in the human body and a naturally occurring free radical scavenger that has been displayed to increase glutathione, regenerates antioxidants $(16,17)$. $\alpha$-LA is a natural antioxidant synthesized in the human body and is an ideal neuroprotective antioxidant that increases acetylcholine production in the pathogenesis of $A D(14,18)$. Supplementation of $\alpha$-LA (which plays a key role in mitochondrial energy production), improves cognitive and learning-remembering functions by increasing total antioxidant capacity and reducing neurodegeneration in the hippocampal region $(18,19)$.

The aim of this study was to evaluate the possible protective effect of donepezil, memantine and $\alpha$-LA combination therapy on the scopolamine-induced rat amnesia model. For this aim, immunoblotting method was used to determine the changes in the expression level of mitochondrial apoptotic proteins, BDNF, M1 and NR2B in the hippocampal and frontal cortex regions.

\section{METHODS}

\subsection{Drugs and Chemicals}

Scopolamine, alpha lipoic acid, donepezil and memantine were purchased from Sigma (St. Louis, USA). Scopolamine and memantine were dissolved in physiological saline (PS) to create a final injection volume of $1 \mathrm{ml} / \mathrm{kg}$. Scopolamine, donepezil, memantine or saline was injected intraperitoneally (i.p) $3 \mathrm{~h}$ before the onset of the cognitive function test (11). All antibodies were supplied from Santa Cruz Biotechnology, Inc (Santa Cruz, CA, USA).

\subsection{Animals and Conditions}

In the present study, 48 healthy Wistar albino male rats, $12-$ 14 weeks old, weighing 250-300 g, supplied from the XXX were used. All procedures related to animal experiments were approved by the Ethics Commission from Experimental Animals Research and Implementation Centre, DEHAMER (App. No: 31.2018.Mar).

All animals were conditioned with the reverse of the circadian light cycle for ten days (12 h light/dark) where they were housed in rooms with controlled temperature and humidity $22 \pm 2^{\circ} \mathrm{C}$ and $40-60 \%$, respectively) before the onset of the behaviour experiments. The rats kept in polypropylene cages (four animals per cage) were accessed to unlimited access to standard rat diet and drinking water.

\subsection{Experimental Design}

Rats were randomly divided into two groups as control and amnesia groups. The rats in the control group were given saline (saline group: $0.9 \%$ Saline) treatment, a-lipoic acid (LA group: $100 \mathrm{mg} / \mathrm{kg}$ ) (20), donepezil + memantine ( $\mathrm{D}+\mathrm{M}$ group: $0.1 \mathrm{mg} / \mathrm{kg}+1 \mathrm{mg} / \mathrm{kg}$; as a positive control) (17) or donepezil + memantine + a-lipoic acid ( $D+M+L A$ group) treatment, while all of the amnesia groups either did not receive treatment (amnesia group: Scopolamine $1 \mathrm{mg} / \mathrm{kg}$, as a negative control) $(21,22)$ or were treated with LA (LA amnesia group: Scopolamine $1 \mathrm{mg} / \mathrm{kg}+$ a-lipoic acid $100 \mathrm{mg} / \mathrm{kg}$ ), D+M (D+M amnesia group: Scopolamine $1 \mathrm{mg} / \mathrm{kg}+$ donepezil $0.1 \mathrm{mg} / \mathrm{kg}$ + memantine $1 \mathrm{mg} / \mathrm{kg}$ ) or $\mathrm{D}+\mathrm{M}+\mathrm{LA}$ ( $\mathrm{D}+\mathrm{M}+\mathrm{LA}$ amnesia group: Scopolamine $1 \mathrm{mg} / \mathrm{kg}+$ donepezil $0.1 \mathrm{mg} / \mathrm{kg}+$ memantine 1 $\mathrm{mg} / \mathrm{kg}+$ a-lipoic acid $100 \mathrm{mg} / \mathrm{kg}$ ). Forty-eight rats were used, six in each group. All treatments were given as i.p. for 14 days. (Fig. 1).

On the 15th day the rats were anesthetized and killed by cervical dislocation. The brain was quickly removed and cleaned with ice-cold $0.1 \mathrm{M}$ phosphate buffer saline (PBS; $\mathrm{pH}$ 7.4). The hippocampus and frontal cortex regions were immediately dissected according to Paxinos rat brain atlas (23) and then all dissected brain regions were stored at -80 ${ }^{\circ} \mathrm{C}$ using in immunoblotting assay. 


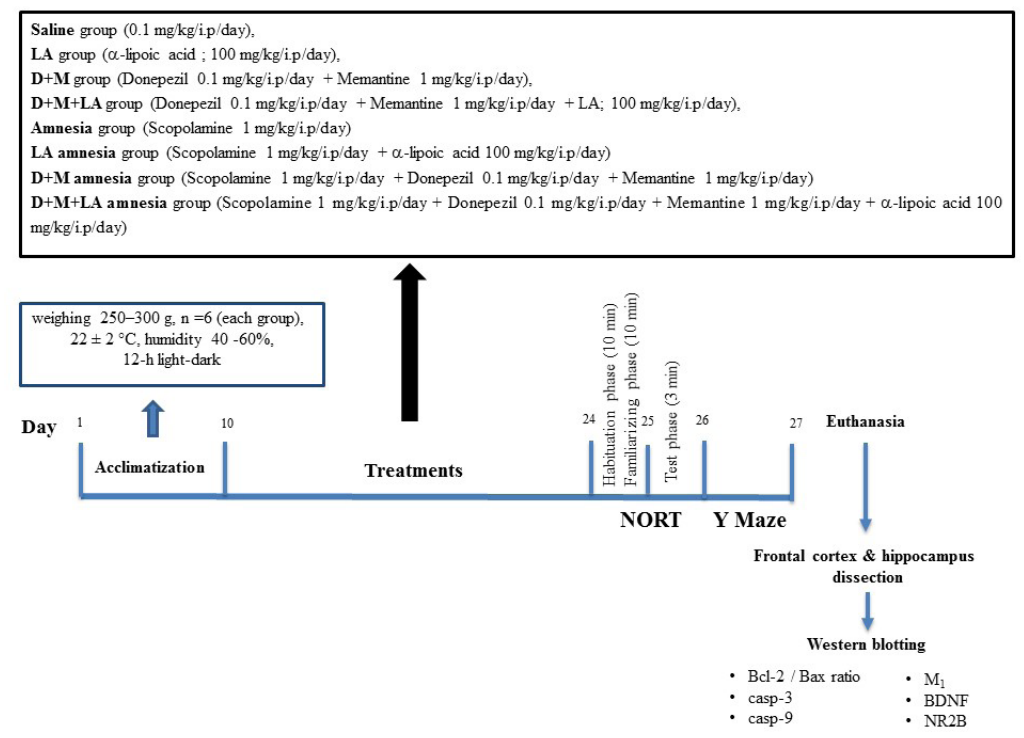

Figure 1. An overview of experimental design

\subsection{The Evaluation of Cognition Functions}

NOR test was used as a measure of short-term/working memory was used to evaluate the cognitive status of rats and the effect of treatments. NORT steps were carried out as follows: Acclimation, Trial 1 and 2. In the acclimation phase that the test equipment does not contain any objects, the rats were accustomed to the test set up for $3 \mathrm{~min}$. In the first trial phase, two identical objects were presented to the rats for 3/10 $\mathrm{min}$. In the second trial phase, which was applied $24 \mathrm{~h}$ after the first trial phase, one of the objects (a familiar object from $1^{\text {st }}$ trial phase) presented was replaced with a new object (a novel object) and presented to the rats for $3 \mathrm{~min}$. The formula described previously was used in the expression of the discrimination index created by measuring the time that the rats spent on investigated each object during Trial 2 (24).

The Y-maze test, a behavioural test that meant to quantify working memory and measures the willingness to discover new environments in rats, was used to evaluate the shortterm spatial memory performance and cognitive deficiencies of the rats and the effects of treatments on cognition (25). Rats in the own cage were placed in the test room $1 \mathrm{~h}$ before the single session $Y$ maze study to minimize the effects of the stress factor on behaviour during the test. The $Y$ maze made of plexiglass material, $15 \mathrm{~cm}$ wide, $40 \mathrm{~cm}$ long, $30 \mathrm{~cm}$ high (three-arm maze with equal angles $\left(120^{\circ}\right)$ between all arms). The rat was placed in the centre of the maze and allowed to move freely through three arms during the test section ( $8 \mathrm{~min}$ ). In the estimating short-term memory, consecutive selections such as $A C B, C A B$, or $B A C$ were recorded. Meanwhile, triad (set of three letters) containing all three letters is scored as alternation. Total number of entries should not include first recorded arm (always $B$ ). The sequence and the number of arm entries were recorded using a video camera for each rat. The four limbs of the rat in the arm were counted as an entry. Successive entry in each arm was considered as alteration in behavior (26). The alternation score (\%) for each rat was indicated as the total arm entries minus two and multiplied by 100 (26).

\subsection{Immunoblotting}

Before transferred onto the membrane (nitrocellulose, Schleicher and Schuell, Germany), all homogenized brain regions were separated into proteins with $12 \%$ SDSPAGE. The membranes were blocked by $1 \%$ BSA in Trisbuffered saline with $0.1 \%$ Tween $20(\mathrm{pH} \mathrm{7.6)}$ for $2 \mathrm{~h}$, and then incubated $14 \mathrm{~h}$ at $4{ }^{\circ} \mathrm{C}$ with the related antibody [BCl-2 (1:100; sc-7382; @ 26 kD), Bax (1:100; sc-20067; @ 23 kD), casp-3 (1:200; sc-56053; @ 20 kD), casp-9 ( 1:200; sc-56076; @ 35 kD), M (1:100; sc-365966; @ 52kD), BDNF (1:200; ab9794; @ 15 kD), or NR2B (1:100; sc-365597; @ 180 kD). Then, all membranes were incubated with a secondary antibody (sc-2354, 1:5000) for $1 \mathrm{~h}$ at $21 \pm 3{ }^{\circ} \mathrm{C}$ temperature. Densitometric analysis of labelled proteins were performed using Bio-Rad Molecular Analyzer software (free edition, www.totallab.com). All values were normalized to b-actin (1:200 dilution; Santa Cruz, CA, USA).

\subsection{Statistical Analysis}

GraphPad software (Prism 3.0; GraphPad Software, San Diego, CA, USA) was used for statistical analysis. All data are represented as mean \pm SEM. The groups of data were compared with analysis repeated measures two-way ANOVA followed by Bonferroni multiple comparison post hoc tests. $p<0.05$ was considered a statistically significant difference between groups. 


\section{RESULTS}

\subsection{Behavioural Parameters}

There was no significant difference in the difference score obtained from the NORT results of the $L A, D+M$ and $D+M+L A$ groups compared to the saline group. According to NOR test results, the difference score was found to be significantly decreased in the amnesia group as compared to the saline group ( $t=4.777, p<0.001 ; d F=56$ ). While the difference score increased in the amnesia groups treated with $D+M$ and $D+M+L A$ compared to the untreated amnesia group $(t=4.143$, $p<0.01 ; t=6.610, p<0.001$, respectively; Fig. $2 A$ ), there was no significant change in the amnesia group treated with LA.

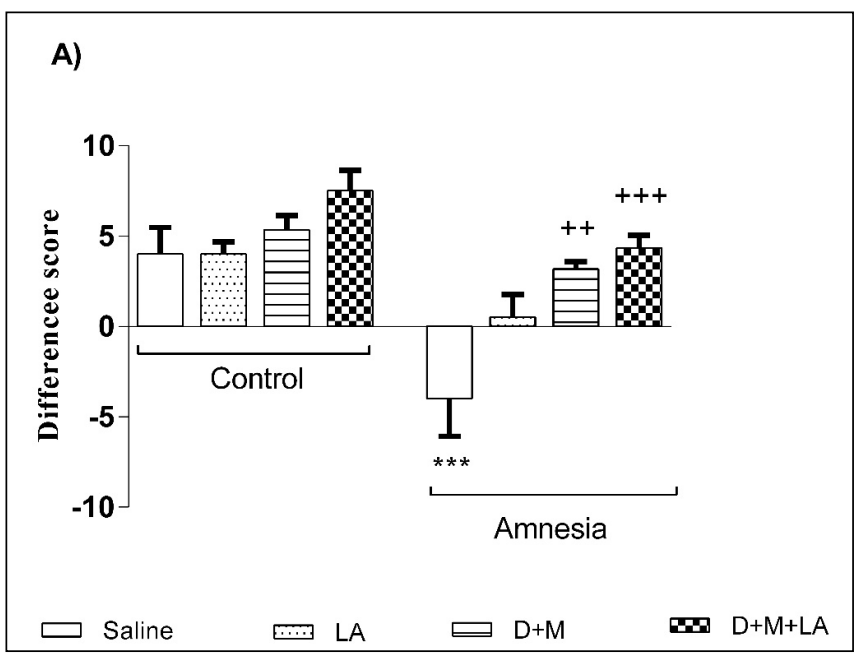

Figure 2. According to a) NORT and b) Y-maze tests, the effect of saline, $\alpha$-lipoic acid $(L A)$, donepezil $(D)+$ Memantine $(M)(D+M)$, $D+M+L A$ combine treatment on the scopolamine-induced amnesia rat cognitive deficit.

All data are represented as mean \pm SEM. ${ }^{* * *} p<0.001$, compared to saline treated-control group; $++p<0.01$ and $+++p<0.001$, compared to only scopolamine group, $p<0.05$ compared to $D+M$-treated scop group $(n=8)$.

There was not detected differently in the mean change behaviour scores for the saline, LA, D+M, and D+M+LA groups were $82.3 \pm 5.4,76.6 \pm 6.9,85.0 \pm 5.8$, and $83.0 \pm$ $5.3 \%$, respectively. The amnesia group showed a significant decrease in the percent of alteration in the behaviour compared to the saline group $(t=5.30, p<0.001, d F=56$; Fig. 2B). It was determined that the percentage of change in behaviour in the $\mathrm{D}+\mathrm{M}$ and $\mathrm{D}+\mathrm{M}+\mathrm{LA}$ amnesia groups improved significantly compared to the amnesia group ( $\mathrm{t}=$ 3.49, $p<0.05 ; t=6.73, p<0.001$; Fig. 2B).

\subsection{Immunoblotting Assay}

Change's expression in the Bcl-2 (anti-apoptotic protein) / Bax (pro-apoptotic protein) ratio, casp-3 and casp-9 levels in the frontal cortex and hippocampus regions were used to evaluate apoptosis in the mitochondrial pathway $(n=4)$. The BDNF and NR2B expression level, contributing to the survival of the central nervous system was used to evaluate neuroplasticity in both brain regions.
There was no statistical difference between the saline group and the treatments administrated to the control group in terms of Bcl-2 / Bax ratio, casp-3, and casp-9 expression levels.

In hippocampal region analysis, it was determined that the $\mathrm{Bcl}-2$ / Bax ratio decreased in the amnesia group compared to the saline group ( $t=6.344, p<0.001$, Fig. $3 A$ ), and the expression levels of casp- 3 and casp- 9 increased ( $t=3.489$, $p<0.05$, Fig. 3C; $t=5.409, p<0.001$, Fig. 3E). It was found that the decrease in $\mathrm{Bcl}-2$ / Bax ratio and the increase in casp-9 almost returned to control (saline group) values with $\mathrm{D}+\mathrm{M}(\mathrm{t}=3.749, \mathrm{p}<0.05 ; \mathrm{t}=7.707, \mathrm{p}<0.001$, respectively) and $\mathrm{D}+\mathrm{M}+\mathrm{LA}(\mathrm{t}=7.497, \mathrm{p}<0.001 ; \mathrm{t}=8.856, \mathrm{p}<0.001$, respectively) treatments administrated to the amnesia group. While casp-3 expression level decreased in amnesia group treated with $\mathrm{D}+\mathrm{M}+\mathrm{LA}$ compared to untreated amnesia group $(\mathrm{t}=4.178$, $\mathrm{p}<0.01$ ), $\mathrm{D}+\mathrm{M}$ treatment applied to amnesia group had no effect on casp-3 expression level. While D+M treatment alone could not reverse the increase in casp-3, adding LA to the combined treatment reversed this situation. In addition, LA did not change the $\mathrm{Bcl}-2$ / Bax ratio and casp-3 expression level in amnesia rats. Differently, it was determined that LA treatment given to the amnesia group caused a decrease in casp-9 expression level in the hippocampus ( $t=5.648, p<$ 0.001).

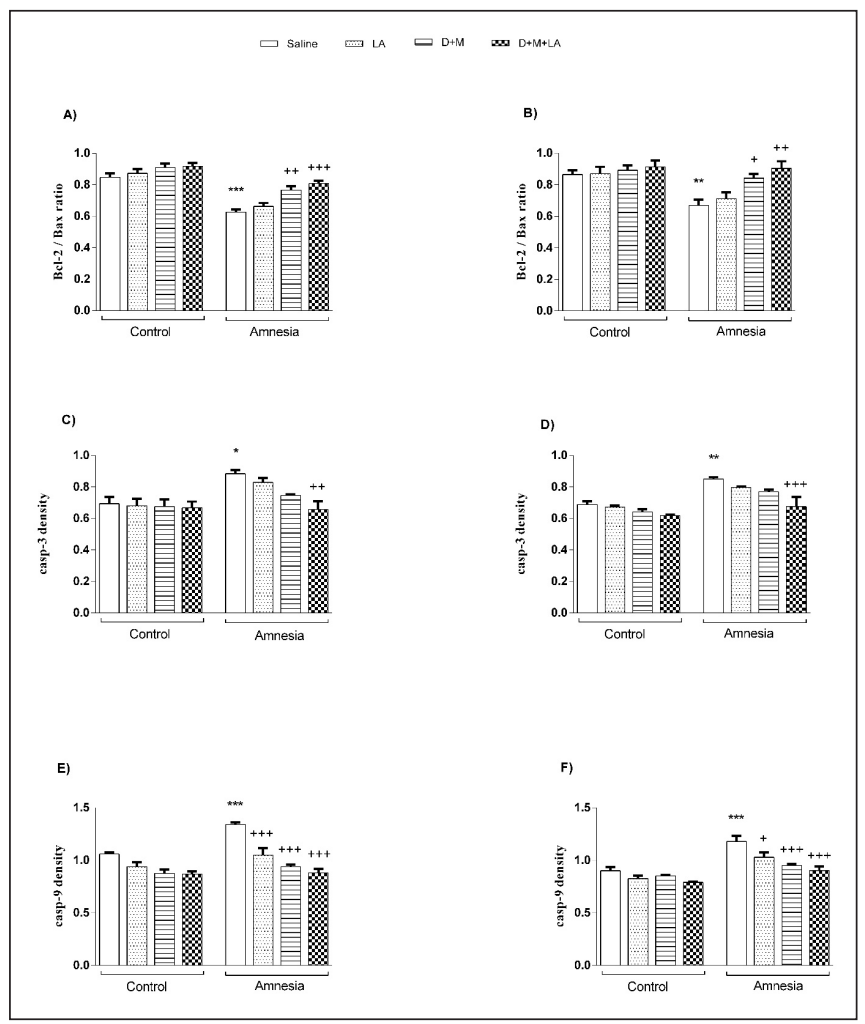

Figure 3. The density of immunoblotting of $a-b)$ Bcl-2 / Bax ratio, $c-d)$ caspase-3, e-f) caspase-9 expression levels of the hippocampus and frontal cortex regions (respectively) of rats in the scopolamineinduced amnesia rat model.

All data are represented as mean \pm SEM. ${ }^{*} p<0.05,{ }^{* *} p<0.01$ and ${ }^{* * *} p<0.001$, compared to saline treated-control group; $+p<0.05$, $++p<0.01$ and $+++p<0.001$, compared to only scopolamine group; $p<0.05$ compared to $D+M$-treated scop group. 
When the amnesia group was compared with the saline group, $\mathrm{Bcl}-2$ / Bax ratio was decreased in the frontal cortex region of the amnesia group compared to the saline group $(t=3.98, p<0.01$, Fig. 3B) but casp-3 and -9 levels were increased ( $t=4.503, p<0.01$, Fig. 3D; $t=5.873, p<0.001$, Fig. $3 \mathrm{~F})$. In the $\mathrm{D}+\mathrm{M}$ amnesia group, $\mathrm{Bcl}-2$ / Bax ratio increased in frontal cortex region, unlike casp-9 expressions $(t=3.23$, $p<0.05 ; t=4.815, p<0.001$, respectively). There was no significant difference in the expression of casp-3 in the $\mathrm{D}+\mathrm{M}$ amnesia group. When the amnesia group treated with $D+M+L A$ was compared with the amnesia group, it was determined that the $\mathrm{Bcl}-2$ / Bax ratio decreased and casp-3 and -9 expressions increased $(t=5.24, p<0.001$; $\mathrm{t}=4.925, \mathrm{p}<0.001 ; \mathrm{t}=5.873, \mathrm{p}<0.001$; respectively). In addition, in the hippocampus region of the amnesia group, $\mathrm{D}+\mathrm{M}+\mathrm{LA}$ treatment increased $\mathrm{BCl}-2$ / Bax ratio compared to $\mathrm{D}+\mathrm{M}$ treatment group $(\mathrm{t}=3.749, \mathrm{p}<0.05)$. LA treatment in amnesia rats did not change the $\mathrm{Bcl}-2$ / Bax ratio and casp-3 level as in the frontal cortex region. It was determined that the treatment given to amnesia group treated with LA caused a decrease in the expression level of casp-9 ( $t$ $=3.174, \mathrm{p}<0.05$ ).

There was no statistical difference between the saline group and the treatments administrated to the control group in terms of M1, BDNF and NR2B expression levels in both regions. It was determined that $M_{1}$ expression levels decreased in the hippocampus and frontal cortex regions in the amnesia group compared to the saline treated-control group ( $t=5.725$, Fig. 4A; $t=6.390$, Fig. 4B; $p<0.001$ in both regions). Among the treatments administrated to amnesia groups, $\mathrm{D}+\mathrm{M}$ and $\mathrm{D}+\mathrm{M}+\mathrm{LA}$ treatments were determined to significantly increase $M_{1}$ expression in the hippocampal region compared to the amnesia group $(t=4.425, p<0.01$; $t=8.242, p<0.001$, respectively). Similar to the hippocampus, it was determined that the $\mathrm{D}+\mathrm{M}$ and $\mathrm{D}+\mathrm{M}+\mathrm{LA}$ treatments administrated to the amnesia group showed the same effect in the frontal cortex region $(t=3.238, p<0.05 ; t=7.080, p<$ 0.001 , respectively).

When the BDNF expression level was examined in the hippocampus region, it was determined that the BDNF level of the amnesia group decreased significantly compared to the saline group ( $t=5.676, p<0.001$, Fig. $4 C$ ). In all groups treated for scopolamine-induced amnesia, BDNF level was found to increase significantly compared to the amnesia group ( $t=3.997, p<0.01$ for $D+M$-treatment; $t=7.182, p<$ 0.001 for $\mathrm{D}+\mathrm{M}+\mathrm{LA}$-treatment). It was determined that the BDNF expression level of the amnesia group in the frontal cortex region decreased compared to the saline group $(t=5.522, p<0.001$, Fig. 4D). It was found that the decrease in BDNF expression was reversed and reached control values with $D+M$, and $D+M+L A$ treatments administered to scopolamine-induced amnesia group $(t=3.654, p<0.05$; $t=6.902, p<0.001$ ).

In both regions, NR2B expression level was increased in the amnesia group compared to the saline group $(t=6.810$, $p<0.001$, Fig. 4E; $t=3.824, p<0.01$, Fig. 4F). In addition, NR2B expression was decreased in the D+M amnesia group compared to the amnesia group ( $t=3.190, p<0.05 ; t=3.364$, $\mathrm{p}<0.05$ ) in the both regions. In the hippocampus and frontal cortex regions, NR2B expression was decreased in the $\mathrm{D}+\mathrm{M}+\mathrm{LA}$ amnesia group compared to the amnesia group $(t=6.781, p<0.001 ; t=6.098, p<0.01)$. While LA treatment administrated to the amnesia group did not cause any change in BDNF and NR2B expressions in both regions, it increased the $M_{1}$ expression level significantly compared to the amnesia group $(p<0.05)$.

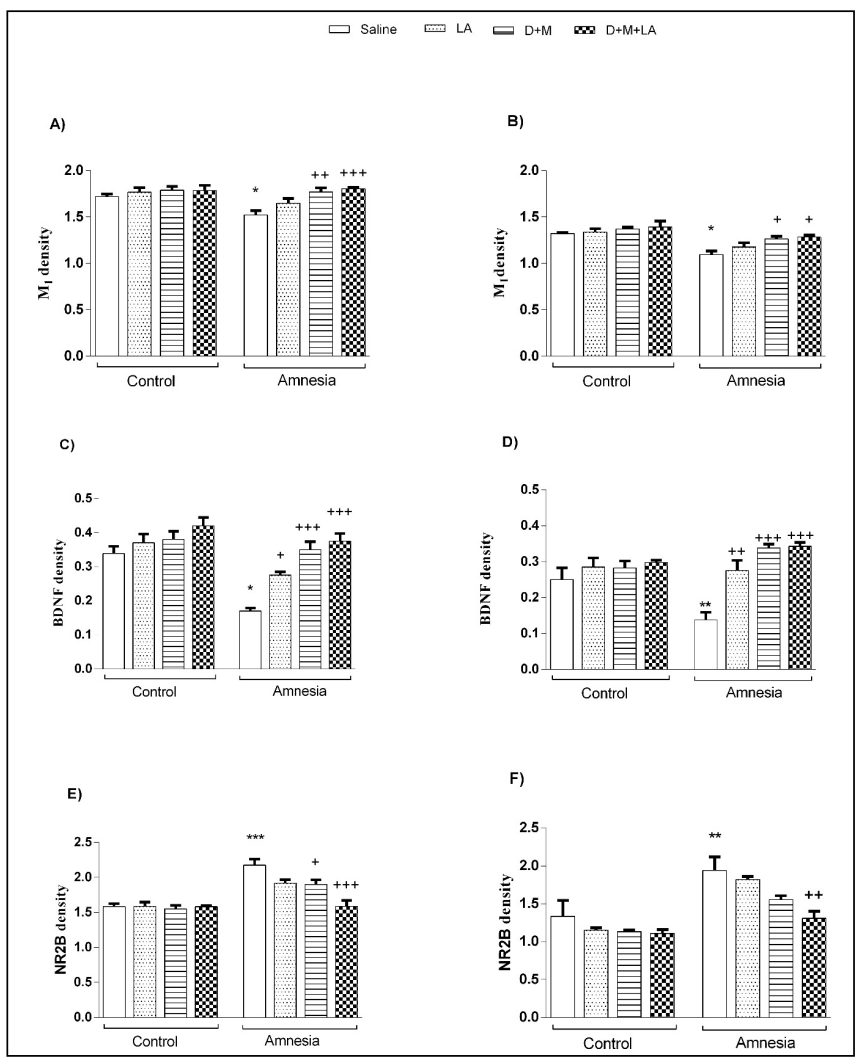

Figure 4. The density of immunoblotting of $\left.a-b) M_{1} c-d\right) B D N F$, e-f) NR2B expression levels of the hippocampus and frontal cortex regions (respectively) of rats in the scopolamine-induced amnesia rat model.

All data are represented as mean \pm SEM. ${ }^{*} p<0.05,{ }^{* *} p<0.01$ and ${ }^{* * *} p<0.001$, compared to saline treated-control group; ${ }^{+} p<0.05$, ${ }^{++} p<0.01$ and ${ }^{+++} p<0.001$, compared to only scopolamine group; $p<0.05$ compared to $D+M$-treated scop group.

$D+M+L A$ treatment in the hippocampus region of the amnesia groups increased $M_{1}$, BDNF and decreased NR2B expression level compared to $\mathrm{D}+\mathrm{M}$ treatment, while the difference between these two treatments in the cortex region was determined only in the BDNF expression level. Representative immunoblotting membranes obtained from different brain tissues for all antibodies used in the experiments were shown in Fig. 5. 


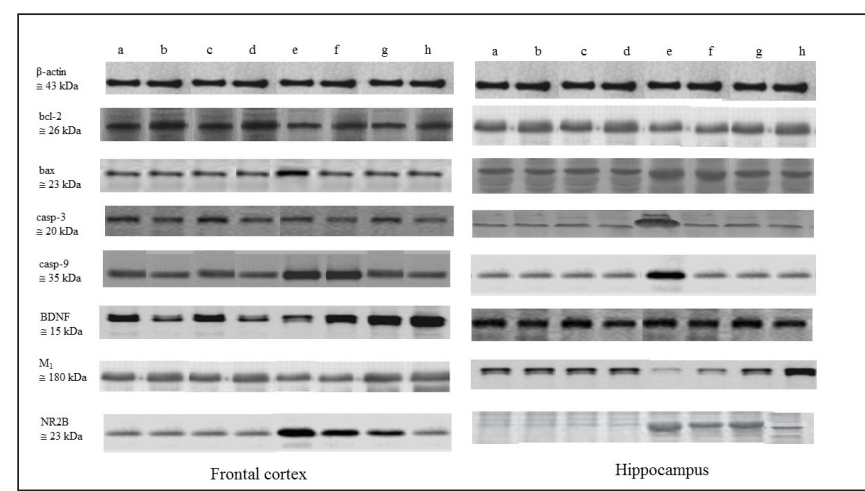

Figure 5. The representative image of membranes obtained from western blotting experiments in the scopolamine-induced amnesia rat model.

a. saline group; $b$. $\alpha$-lipoic acid group; c. Donepezil + Memantine group; $d$. Donepezil + Memantine $+\alpha$-lipoic acid group; e. amnesia (scopolamine-induced) group; f. a-lipoic acid amnesia group, $g$. Donepezil + Memantine amnesia group; $h$. Donepezil + Memantine $+\alpha$-lipoic acid amnesia group.

\section{DISCUSSION}

The main findings of the current study demonstrated that combined treatment of memantine, donepezil and $\alpha$-LA ameliorated behavioural and memory impairment in rats in the scopolamine-induced amnesia model. We have found that scopolamine-treated rats showed cognitive dysfunction in both the Y-maze and NOR test, while these decreased cognitive functions were reversed with memantine, donepezil and a-LA combined treatment. In addition, it was determined that the decreased $\mathrm{Bcl}-2$ / Bax ratio and increased casp-3 and -9 expression levels caused by scopolamine via the combined treatment in the hippocampus and frontal cortex regions were also improved. Another finding of the study is that decreased BDNF, $M_{1}$ and increased NR2B expressions due to amnesia in both cortex and hippocampus regions were reversed with combined treatment.

Previous studies reporting that $\alpha$-LA plays a role in the biosynthesis of mitochondrial matrix proteins highlight its positive effect on mitochondria. $\alpha$-LA performs indirect effect on mitochondria, which also has a protective role in the context of neuronal damage in the process of apoptosis, by stimulating mitochondrial biogenesis (14). In a study evaluating the effect of the expression of the $\mathrm{Bcl}-2$ apoptosis regulator in the hippocampal region of rats, it was determined that $\mathrm{Bcl}-2$ expression was increased due to $\alpha$-LA administration (27). Since AD and amnesia are known to be related to mitochondrial damage occurring in neurons, some of the researches for the treatment of AD have turned to the search for a strong mitochondrial therapeutic agent. In the study that illuminated the neuroprotective molecular mechanism of $\alpha$-LA to $\mathrm{H} 2 \mathrm{O} 2$-induced cell death, $\mathrm{H}_{2} \mathrm{O}_{2}$ exposure has been reported to cause a decrease in the ratio of $\mathrm{Bcl}-2$ / Bax that supports apoptosis and this is prevented by a-LA therapy. The results of this study also reported that suppression of casp-3 activation suppressed the formation of apoptosome (19). The improvements in cognitive and behavioural performances by $\alpha$-LA observed here are in agreement with previous reports and our results suggest that $\alpha$-LA might play a role in regulating the development of apoptosis. Therefore, $\alpha$-LA may act as a potential therapeutic agent for its beneficial effect on the pathogenesis of amnesia. Our study results indicate that the combined treatment of memantine, donepezil and $\alpha$-LA may be effective in mitochondria-mediated apoptosis. We suggest that $\alpha$-LA may be effective in the treatment of amnesia due to its potential to restore mitochondrial damage, in addition to the treatment of memantine and donepezil in the clinic from our study findings.

There are studies in the literature that use $\alpha$-LA therapy with various agents acting on central or peripheral nerve cells. In the schizophrenia model induced by ketamine, in the study in which the effect of $\alpha$-LA and clozapine combined therapy was evaluated with Y-maze, it was found that combined therapy could restore impaired behavioural parameters. In the same study results, it has been reported that BDNF deficiency in frontal cortex caused by ketamine-induced is significantly reversed by $\alpha$-LA and clozapine combined treatment (28). Although the relationship between $A D$ and $B D N F$ is still controversial, many data indicate that there is a negative correlation. Studies suggest that the decrease of BDNF mRNA in the hippocampus of individuals with $A D$ suggests that BDNF may contribute to the progression of cell loss in AD. Peng et al. (2009) found that significant deficiency in BDNF levels in patients with $A D$ showed a positive correlation with the degree of cognitive impairment of AD (29). In human and animal studies using donepezil in the treatment of $A D$, the drug has been shown to increase the level and expression of BDNF (30).

In addition to their vital role in synaptic transmission and plasticity, NMDARs also play a critical role in the neuronal survival pathway. Especially, synaptic NMDAR activation increases cell survival, while extra-synaptic activation triggers cell death. The impaired balance between these two NMDA population groups causes chronic neurodegenerative diseases (31). Current studies provide documents on NMDA receptor binding in $A D$, but details of changes in specific NMDA receptor subunits are not sufficient. Although studies in the literature show that NMDA receptors play a role in the pathogenesis of $A D$, the change in the expression levels of NMDA receptors and their relationship with apoptosis has not been clearly determined in this process. As in the results of the study by Liu et al., (2012) in our results high NR2B expression and apoptosis showed remarkable harmony in the hippocampus and frontal cortex regions of AD-like rats. The effects of acute donepezil or memantine or the combined application of both treatments on memory performances were tested in studies conducted to evaluate the treatments used to prevent the stimulant effect of NMDAR and to increase cholinergic function $(32,33)$. Memantine, a widely accepted NMDA antagonist for treating $A D$, has also been proven to have an enhancing effect on the BDNF level (33). While some data do not fully support the positive correlation 
between the BDNF and AD, it is unlikely to reject that higher BDNF levels actually improve cognitive dysfunction in $A D$. There are still problems in the development of BDNF-based therapies as BDNF increases in the early stage of $A D$ and decreases in the final stage of $A D(6)$. Our results have been shown to increase BDNF expression in both hippocampus and frontal cortex with combined therapy in accordance with the literature. Moreover, in our findings, the increase in NR2B expression and the decrease in bcl-2/bax ratio due to scopolamine-induced was reversed with $\alpha$-LA treatment.

The association of acetylcholine with memory and cognitive function is well documented in the literature $(34,35)$. Most of this information is about that the various improvements in cognitive function can be observed after cholinergic receptor activation. A role for $\mathrm{mAChR}$ has been hypothesized in many neurodegenerative disorders such as post-traumatic stress disorder. The most common types of the mAChRs that are found in the brain are $M_{1}$ type receptors and they are localized in the different brain regions such as hippocampus, cortex, and striatum (36). mAChRs are known to play a role in learning and memory, but the clarification of the precise roles of these receptors remains. It has been reported that $M_{1}$ blockage with different antagonists causes cognitive reductions in attention, learning, and memory. Muscarinic antagonists are an interesting option for inducing cognitive impairment and finding new pharmacological alternatives (37). Especially, the effect of scopolamine is interpreted as a cholinergic deficiency, which emphasizes the effect and importance of acetylcholine on memory functions (5). As a result of the study conducted by Araújo et al. (2017), it was emphasized that a-LA increases muscarinic $M_{1}$ receptor affinity in the hippocampus and frontal cortex regions (38). Therefore, in this study, the change in the expression of the $M_{1}$ subtype of mAChRs, which has an important role in cognitive functions and behavioural parameters, was evaluated by adding $\alpha$-LA to the combination of donepezil and memantine used in the routine treatment of AD. In the study in which $\alpha$-LA therapy was evaluated in the memory deficits in mice, it was determined that the treatment increased muscarinic $M_{1}$ receptor expression in the hippocampus (39). According to our findings, it was determined that the administration of $\alpha$-LA to combined donepezil and memantine therapy in the hippocampus and frontal cortex regions increased the decreased $M_{1}$ receptor expression in scopolamineinduced amnesia rat model. In both our behavioural tests and molecular experimental results, the addition of $\alpha$-LA to combined therapy showed that it significantly improved memory and the level of $M_{1}$ protein expression. As emphasized at the end of the related study, our results suggest that a-LA may have therapeutic potential in improving cholinergic functions. The down regulation of $M_{1}$ receptors may be regarded as a natural consequence of the plasticity. Therefore, this result may suggest that cholinergic receptors may mediate the improvement of cognitive functions in the $A D$ process induced by scopolamine as a result of the addition of $\alpha$-LA to AD routine treatment. Our study results suggested administration of $\alpha$-LA to the combination of donepezil and memantine therapy as an excellent therapeutic candidate for cholinergic hypofunction in neurodegenerative disorders such as amnesia.

\section{CONCLUSION}

According to the results of our study, it has been shown that muscarinic $\left(M_{1}\right)$ and glutamatergic (NR2B) systems play an important role in Alzheimer's pathogenesis. $\alpha$-LA acid is a powerful antioxidant. Although it corrects apoptosis accompanied by oxidative stress, its effect alone is insufficient since it does not have as strong effects on the muscarinic and glutamatergic systems as other drugs. At present, donepezil and memantine are often preferred for treatment of AD based on cognitive deficits. We determined that the antiapoptotic effect of adding $\alpha$-LA to donepezil and memantine treatment improves cognitive functions by regulating changes in $M_{1}$, NR2B and BDNF protein expressions in AD. In further studies, different studies and clinical evaluations can be pioneered by evaluating the effects of the drugs used in the treatment on the side effect profiles.

Conflict of interest: We certify that there is no conflict of interest with any financial, personal, or other relationships with other people or organizations related to the material discussed in the manuscript.

Disclosure of interest: The authors report no conflict of interest

\section{REFERENCES}

[1] Wang R, Reddy PH. Role of glutamate and NMDA receptors in Alzheimer's disease. J Alzheimer's Dis 2017; 57:1041-1048.

[2] Schliebs R, Arendt T. The cholinergic system in aging and neuronal degeneration. Behav Brain Res 2011; 221:555-563.

[3] Falsafi SK, Deli A, Höger H, Pollak A, Lubec G. Scopolamine administration modulates muscarinic, nicotinic and NMDA receptor systems. PLoS One 2012; 7:e32082: 10.1371/journal. pone.0032082

[4] Volpicelli LA, Levey Al. Muscarinic acetylcholine receptor subtypes in cerebral cortex and hippocampus. Prog Brain Res 2004; 145:59-66.

[5] Weinstein G, Beiser AS, Choi SH, Preis SR, Chen TC, Vorgas D. Serum brain-derived neurotrophic factor and the risk for dementia: the Framingham Heart Study. JAMA Neurology 2014; 71:55-61.

[6] Song JH, Yu JT, Tan L. Brain-derived neurotrophic factor in Alzheimer's Disease: Risk, mechanisms, and therapy. Mol Neurobiol 2015; 52:1477-1493.

[7] Mishizen-Eber AJ, Robert A, Rissman RA, Ikonomovic MD, Wolfe BB, Armstrong DM. Biochemical and molecular studies of NMDA receptor subunits NR1/ $2 A / 2 B$ in hippocampal subregions throughout progression of Alzheimer's Disease pathology. Neurobiol Dis 2004; 15:80-92.

[8] Liu J, Chang L, Song Y, Li H, Wu Y. The role of NMDA receptors in Alzheimer's Disease. Front Neurosci 2019; 13:43.

[9] Ota H, Ogawa S, Ouchi Y, Akishita M. Protective effects of NMDA receptor antagonist, memantine, against senescence 
of PC12 cells: A possible role of nNOS and combined effects with donepezil. Exp Gerontol 2015; 72:109-116.

[10] Liang YQ, Tang XC. Comparative effects of huperzine A, donepezil and rivastigmine on cortical acetylcholine level and acetylcholinesterase activity in rats. Neurosci Let 2004; 361:56-59.

[11] Rahman A, Lamberty Y, Schenker E, Cella M, Languille S, Bordet R. Effects of acute administration of donepezil or memantine on sleep-deprivation-induced spatial memory deficit in young and aged non-human primate grey mouse lemurs (Microcebus murinus). Plos One 2017; 12:e0184822.

[12] Li Q, Chen M, Liu H, Yang G Yang L. Expression of APP, BACE1, $A C h E$ and ChAT in an AD model in rats and the effect of donepezil hydrochloride treatment. Mol Med Rep 2012; 6:1450-1454.

[13] Raina P, Santaguida P, Ismaila A, Patterson C, Cowan D, Lewine $M$. Effectiveness of cholinesterase inhibitors and memantine for treating dementia: evidence review for a clinical practice guideline. Ann Inter Med 2008; 148:379-397.

[14] Dos Santos SM, Romeiro CFR, Rodrigues CA, Cerqueira ARL, Monteiro MC. Mitochondrial dysfunction and alpha-lipoic acid: Beneficial or harmful in Alzheimer's Disease? Oxid Med Cell Longev 2019; 8409329.

[15] Khalili M, Eghtesadi S, Mirshafiey A, Eskandari G, Sanoobar M, Sahraian MA. Effect of lipoic acid consumption on oxidative stress among multiple sclerosis patients: a randomized controlled clinical trial. Nutr Neurosci 2014; 17(1):16-20.

[16] Martin DS, Towey M, Horrobin DF, Lynch MA. A diet enriched in $\alpha$-lipoic acid reverses the age-related compromise in antioxidant defences in rat cortical tissue. Nutr Neurosci 2000; 3(3):193-206.

[17] de Sousa CNS, da Silva Leite CMG, da Silva Medeiros I, Vasconcelos LC, Cabral LM, Patrocínio CFV. Alpha-lipoic acid in the treatment of psychiatric and neurological disorders: a systematic review. Metab Brain Dis 2019; 34(1):39-52.

[18] Zhang YH, Wang DW, Xu SF, Zhang S, Fan YG, Yang YY. $\alpha$-Lipoic acid improves abnormal behavior by mitigation of oxidative stress, inflammation, ferroptosis, and tauopathy in P301S Tau transgenic mice. Redox Biol 2018; 14:535-548.

[19] Kamarudin MN, Mohd Raflee NA, Hussein SS, Lo JY, Supriady $H$, Abdul Kadir H. (R)-(+)- $\alpha$-lipoic acid protected NG108-15 cells against $\mathrm{H}_{2} \mathrm{O}_{2}$-induced cell death through PI3K-Akt/GSK-3 $\beta$ pathway and suppression of NF-k $\beta$-cytokines. Drug Des Devel Ther 2014; 8:1765-1780.

[20] Sehirli AO, Aksoy U, Kermeoglu F, Kalender A, Savtekin G, Ozkayalar $\mathrm{H}$. Protective effect of alpha-lipoic acid against apical periodontitis-induced cardiac injury in rats. Eur J Oral Sci 2019; 127:333-339.

[21] Aykac A, Ozbeyli D, Uncu M, Ertaş B, Kılınç O, Şen A. Evaluation of the protective effect of Myrtus communis in scopolamineinduced Alzheimer model through cholinergic receptors. Gene 2019; 689:194-201.

[22] Mohammadpour T, Hosseini M, Naderi A, Karami R, Sadeghnia HR, Soukhtanloo M, Vafaee F. Protection against brain tissues oxidative damage as a possible mechanism for the beneficial effects of Rosa damascena hydroalcoholic extract on scopolamine induced memory impairment in rats. Nutr Neurosci 2015; 18(7):329-336.

[23] Paxinos G and Watson C. (1986). The rat brain in stereotaxic coordinates. 2nd ed. London: Academic Press.
[24] Mathiasen JR, DiCamillo A. Novel object recognition in the rat: a facile assay for cognition function. Animal model of Diseases. Cur Protoc Pharmacol 2010; 5.59.1-5.59.19.

[25] Wahl D, Coogan SC, Solon-Biet SM, de Cabo R, Haran JB, Raubenheimer D. Cognitive and behavioral evaluation of nutritional interventions in rodent models of brain aging and dementia. Clin Interv Aging 2017; 12:1419-1428.

[26] Lee S, Park HJ, Jeon SJ, Kim E, Lee HE, Kim H. Cognitive Ameliorating Effect of Acanthopanax koreanum Against Scopolamine-Induced Memory Impairment in Mice. Phytother Res 2017; 31:425-432.

[27] Dixit S, Dhar P, Mehra RD. Alpha lipoic acid (ALA) modulates expression of apoptosis associated proteins in hippocampus of rats exposed during postnatal period to sodium arsenite (NaAsO2). Toxicol Reports 2015; 2:78-87.

[28] Vasconcelos GS, Ximenes NC, de Sousa CN, Oliveira Tde $Q$, Lima LL, de Lucena DF. Alpha-lipoic acid alone and combined with clozapine reverses schizophrenia-like symptoms induced by ketamine in mice: participation of antioxidant, nitrergic and neurotrophic mechanisms. Schizophr Res 2015; 165:163-170.

[29] Peng S, Garzon DJ, Marchese M, Klein W, Ginsberg SD, Francis $B M$. Decreased brain-derived neurotrophic factor depends on amyloid aggregation state in transgenic mouse models of Alzheimer's disease. J Neurosci 2009; 29:9321-9329.

[30] Sakr HF, Khalil KI, Hussein AM, Zaki MS, Eid RA, Alkhateeb $M$. Effect of dehydroepiandrosterone (DHEA) on memory and brain derived neurotrophic factor (BDNF) in a rat model of vascular dementia. J Physiol Pharmacol 2014; 65:41-53.

[31] Leveille F, El Gaamouch F, Gouix E, Lecocq M, Lobner D, Nicole $O$. Neuronal viability is controlled by a functional relation between synaptic and extrasynaptic NMDA receptors. FASEB J 2008; 22:4258-4271.

[32] Ihalainen J, Sarajarvi T, Rasmusson D, Kemppainen S, KeskiRahkonen $\mathrm{P}$, Lehtonen $\mathrm{M}$. Effects of memantine and donepezil on cortical and hippocampal acetylcholine levels and object recognition memory in rats. Neuropharmacol 2011; 61:891899.

[33] Wu HM, Tzeng NS, Qian L, Wei SJ, Hu X, Chen SH. Novel neuroprotective mechanisms of memantine: increase in neurotrophic factor release from astroglia and antiinflammation by preventing microglial activation. Neuropsychopharmacol 2009; 34:2344-2357.

[34] Aykac A, Aydın B, Cabadak H, Gören MZ. The change in muscarinic receptor subtypes in different brain regions of rats treated with fluoxetine or propranolol in a Post-traumatic stress disorder model. Behav Brain Res 2012; 232:124-129.

[35] Pepeu G, Giovannini M. The fate of the brain cholinergic neurons in neurodegenerative diseases. Brain Res 2017; 1670:173-184.

[36] Flynn DD, Ferrari-DiLeo G, Mash DC, Levy Al. Differential regulation of molecular subtypes of muscarinic receptors in Alzheimer's disease. J Neurochem 1995; 64:1888-1891.

[37] Soares JC, Fornari, Oliveira MG. Role of muscarinic M1 receptors in inhibitory avoidance and contextual fear conditioning. Neurobiol Learn Mem 2006; 86:188-196.

[38] de Araújo DP, Camboim TGM, Silva APM, Silva CDF, de Sousa RC, Barbosa MDA. Behavioral and neurochemical effects of alpha lipoic acid associated with omega-3 in tardive dyskinesia induced by chronic haloperidol in rats. Can J Physiol Pharmacol 2017; 7:837-843. 
[39] Mahboob A, Farhat SM, Iqbal G, Babar MM, Zaidi NU, Nabavi SM. Alpha-lipoic acid-mediated activation of muscarinic receptors improves hippocampus - and amygdala-dependent memory. Brain Res Bul 2016; 122:19-28.

How to cite this article: Aykac A, Ozbeyli D, Pekol G, Sehirli AO. Evaluation of the Effects of Donepezil, Memantine and $\alpha$-lipoic Acid Combined Administration in Amnesia Rats on Impaired Cognitive Functions in Terms of Behavioural, Apoptotic, Cholinergic and Glutamatergic Systems. Clin Exp Health Sci 2021; 11: 733-741. DOI: 10.33808/clinexphealthsci.856459 